\title{
The TUS space photodetector relative calibration in flight
}

\section{Y. Sagan ${ }^{1}$}

1. Joint Institute for Nuclear Research, Dubna, 141980

2. Dubna State University, Dubna, 141980

E-mail: yaroslav_sagan@mail.ru

\section{Lavrova}

Joint Institute for Nuclear Research, Dubna, 141980, Russia

E-mail: Iavrova@jinr.ru

\section{A. Grinuyk}

Joint Institute for Nuclear Research, Dubna, 141980, Russia E-mail: andrei_grinyuk@mail.ru

\section{A. Tkachenko}

Joint Institute for Nuclear Research, Dubna, 141980, Russia

E-mail:avt@jinr.ru

\section{Tkachev}

1. Joint Institute for Nuclear Research, Dubna, 141980

2. Dubna State University, Dubna, 141980

E-mail:tkatchev@jinr.ru

The TUS experiment is aimed to study the energy spectrum and arrival direction of Ultra High Energy Cosmic Rays at $\mathrm{E}>100 \mathrm{EeV}$ from the space orbit by measuring the fluorescence radiation of the Extensive Atmospheric Shower in the atmosphere. It is the first orbital telescope aimed for such measurements and is taking data since April 28, 2016. During the first turns of operation $20 \%$ PMTs were broken due to HV tuning system failure. For the same reason, the properties of the remaining PMTs are changed. Relative calibration of PMT gains in flight was done and presented based on analyzing TUS background data itself. A reconstruction of EAS arrival directions is done using the relative calibration coefficients.

36th International Cosmic Ray Conference -ICRC2019-

July 24th - August 1st, 2019

Madison, WI, U.S.A.

\footnotetext{
${ }^{1}$ Speaker
} 


\section{Introduction}

The measurements of Cosmic Ray (CR) energy spectrum, composition and arrival directions in the wide energy interval are an important part of modern particle physics and astrophysics. The TUS project's goal is the experimental study of Ultra High Energy Cosmic Rays (UHECR) at energies about $\sim 10^{20} \mathrm{eV}$. The fluorescent and Cherenkov radiation of the EAS generated by UHECR particles should be detected in the Earth's atmosphere on the night side of the orbit at altitudes 400-500 km. An important advantage of space detector is the possibility of taking data from all arrival directions of the sky with the same apparatus and with the same systematic uncertainties. TUS is the first attempt to detect UHECR from space and is a pathfinder for the next more powerful detectors KLYPVE [1] and JEM-EUSO [2].

\section{The TUS detector}

The TUS detector on board Lomonosov satellite was launched into the orbit on April 28, 2016. It has a sun-synchronous orbit with an inclination of $\sim 97^{\circ}$, a period of circulation $\sim 94 \mathrm{~min}$, and height about $470-500 \mathrm{~km}$. After a few months of flight tests and tuning of TUS apparatus on the satellite, the regular data taking have been started. During regular operation, the detector measures the UV background level and adjusts the HV and the sensitivity of the PMTs at the background radiation variation [3]. The TUS detector is presented in Fig.1 and consists of two main parts: a modular Fresnel mirror-concentrator and a photo-receiver matrix with 16x16 Hamamatsu R1463 PMT pixels and the corresponding DAQ electronics. TUS optical system field of view (FoV) is $\pm 4.5^{\circ}$, one pixel FoV is $\sim 10^{-4} \mathrm{sr}$, a PMT quantum efficiency is $\sim 20 \%$ for the wavelengths of $300-400 \mathrm{~nm}$.

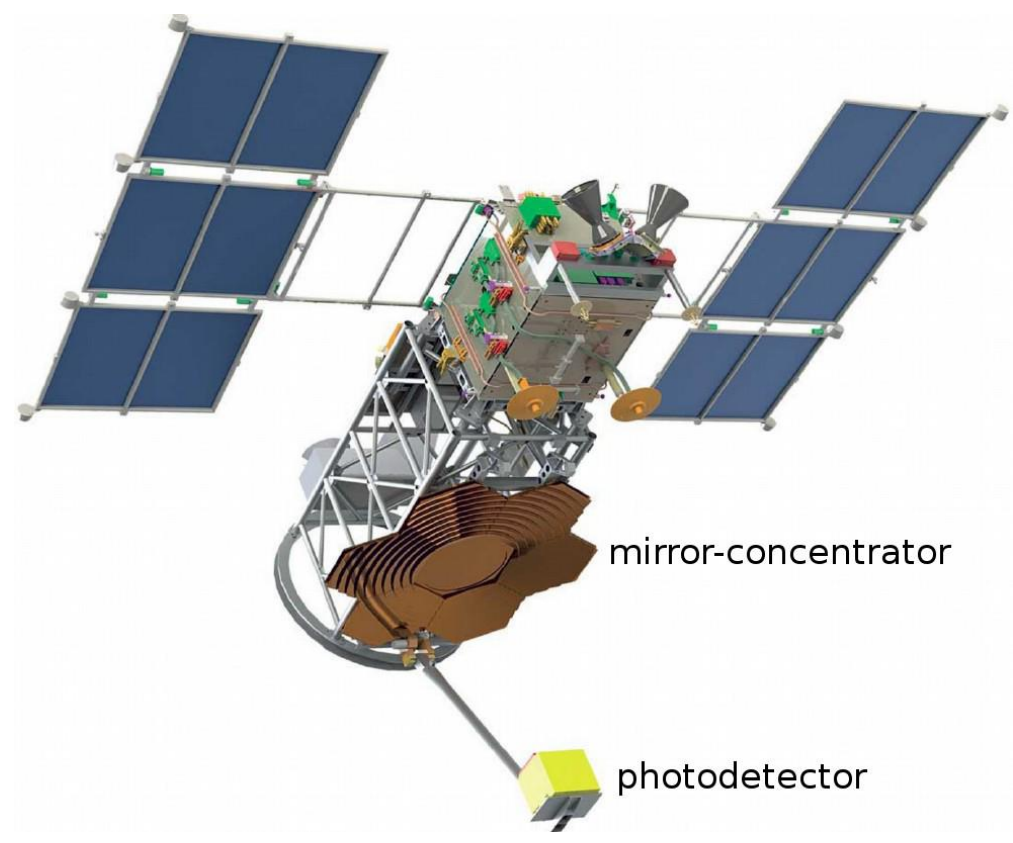

Figure 1: Schematic view of the TUS detector on-board the Lomonosov satellite with the mirror-concentrator and the photo-detector.

The TUS detector has a two-level trigger. The first-level trigger is a threshold trigger: the photo-detector modules board calculates a moving sum of PMT signals during 16 time steps in 
each channel and looks for a moving sum value above a threshold level. The second-level trigger is a pixel-mapping trigger. This procedure selects cases of sequential triggering of spatially contiguous active pixels that are also adjacent in time, allowing for the selection of events with a special spatial-temporal pattern. The TUS DAQ electronics forms a file of 256 time steps for each 256 channels and can operate in four modes intended for detecting various fast optical phenomena in the atmosphere on different time scales. In EAS mode the DAQ electronics works with a time step $\Delta \mathrm{t}=0.8 \mu \mathrm{s}$. The TUS trigger operation is described in more details in [4]. During two years of operation, the TUS detector has measured more than 200000 events most of which are background events. More information about the different types of the background events may be found in $[5]$.

\section{The relative PMT calibration}

During the first days of operation 20\% PMTs were broken due to HV tuning system failure. For the same reason, the properties of the remaining PMTs are changed. Calibration of PMT gains was done based on analyzing background data itself. For this purpose all time sequences (256 time steps) in all pixels (224 working pixels) of all received events were classified into 2 categories: pure "background" pixels and possibly

3 containing signal of any origin. Classification was done by applying multiple cuts on the statistical properties of data of each time sequence. Most important of the cuts are kurtosis, a cut on the slope of the average and cuts on anomalously high and anomalously low mean. Comparison with Monte-Carlo simulated signals shows that the tails of above distributions of real data are significantly wider than what would be expected from pure Poisson based background signal. Therefore, the tails are presumed to contain non-background signals and central part to not contain significant contamination to background.

First extreme values of signal in single time bin not accompanied by high values in adjacent time bins were excluded. This is known to be caused by cosmic rays directly interacting with the detector and the signal sufficiently outside of the time of interaction is not affected by it and can be used. Next relatively slowly increasing (or more rarely decreasing) average value in a pixel makes that pixel excluded. There are known atmospheric effects that are causing such behavior in either the whole detector or just some of the pixels. Then the kurtosis and skewness of amplitude distribution in time is compared to that of Poisson distribution and pixels which don't look like containing Poisson signal are excluded. This effectively excludes pixels where there is even small flash of light. Next - pixels with very high average signal are excluded. Stable in time high signal can happen when flying over bright regions of anthropic origin. Last - some pixels sometimes contain significantly lower average value and are presumed to just not be working correctly and are excluded.

Only events where there were more than 16 "background" pixels (and therefore more than one PMT module) were included in the following steps. Selected in the above way "background" pixels had their mean signal divided by the average mean signal over all "background" pixels in the same event. That ratio was then averaged for that pixel over all the events. The physical background varies in time from event to event across the whole field of view of TUS. Additionally, it varies in each event from pixel to pixel due to ground albedo inhomogeneity and clouds. The above order of averaging was chosen so that it should converge under such conditions to real relative PMT gains Grel, given enough data. Division of data into 3 fully statistically independent 
samples, corresponding to 6 month of operation each, provides consistently repeating coefficients as it presented in Figure 2 that validate this assumption.

It is expected that this method could introduce systematic bias for pixels with extremely high or extremely low PMT gains. Their signals might get counted at the edge of some of the 4 statistical cuts part of the time. This may lead to their gains evaluated as more moderate than they really are. To counter that it may be sufficient to mark calibration of several pixels as unreliable (though pixels with very low gains were already not considered reliable anyway) and their exclusion from event reconstruction.
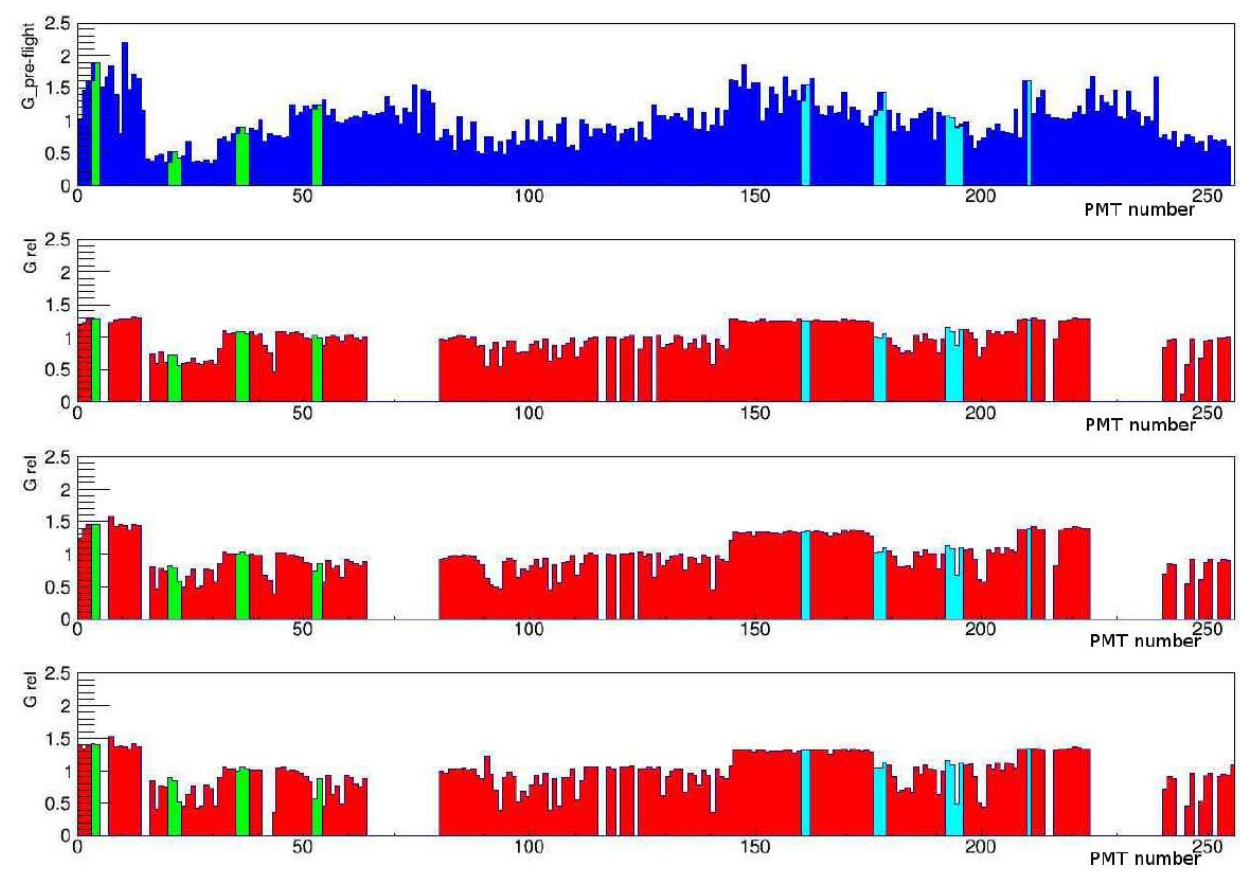

Figure 2: Relative PMT gain coefficients for all 256 channels according to pre-flight measurements (top) and reconstructed from background data for first 3 half-years of operation.

The average value of the absolute relative difference of the PMT coefficients was performed to minimize according to the value of the scale factor for all coefficients. They differ by 0.09 , difference from preflight measurements $\approx 0.35$. Since these coefficients are approximately normalized by one, it can be said that 0.09 is the error of this relative calibration method.

\section{4. $\quad$ EAS candidates}

A few of EAS candidates were found and analyzed according to the understanding of the EAS physics and the TUS detector simulation. The program package was elaborated for TUS events analysis [4]. Some details of the analysis criteria may be found in [6]. 

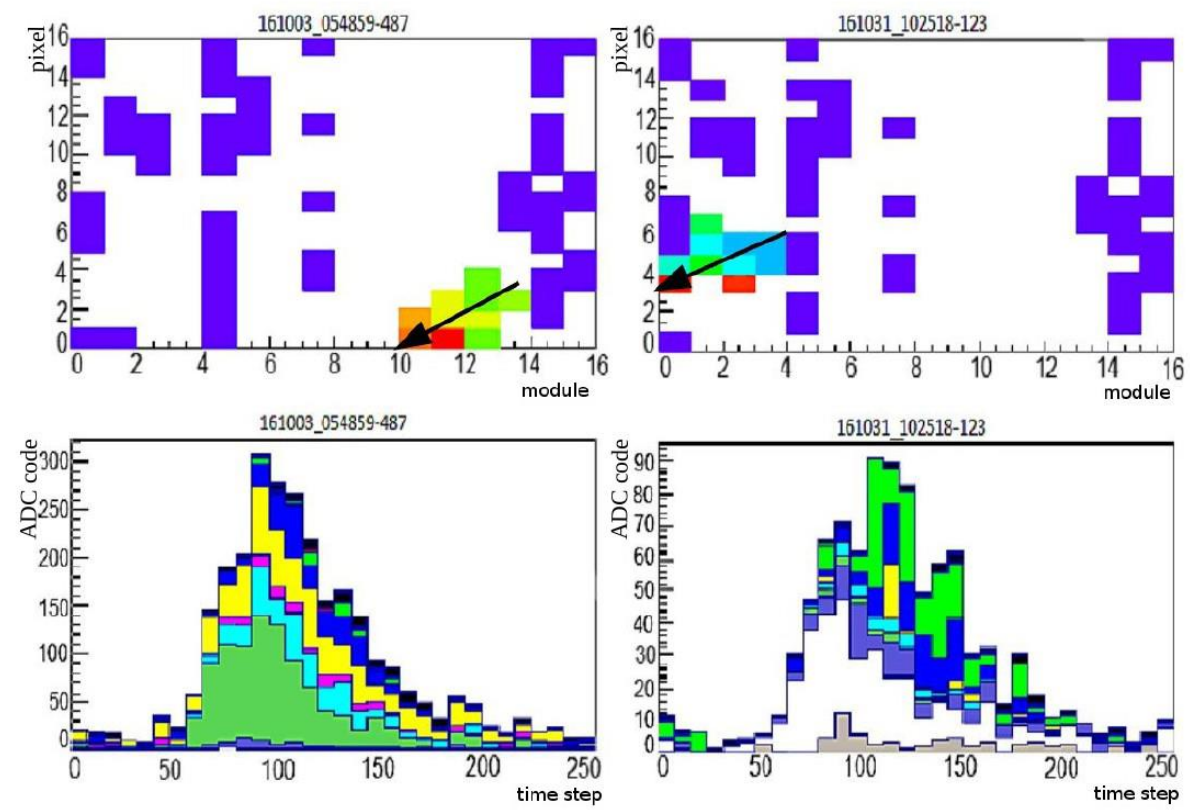

Figure 3: The EAS candidates. Upper plots: image of event with hit pixels and not-working (blue) ones. Bottom plots: the amplitude variation of time for selected hit pixels

In Figure 3 the summary of the events is shown: the date and UTC time of the event measurement are the figure title, hit pixels together with dead ones in the upper panel and the amplitude variation (ADC codes) of time for selected hit pixels in the lower panel. The markers and colors of the selected pixels on the upper panel indicate the arrival times for the maximum EAS signals in the time steps. Active pixels are grouped in an oblong spot. It can be seen from the waveforms that characteristic duration of the signal is $70-100 \mu$ s which is more that one can expect from a vertical EAS. The time position of the maximum of the signal in each pixel has some shift from one pixel to another. This is an argument in favour of the EAS origin for these events. There is a general property of waveforms of two EAS events: the EAS signal starts from dead module then moves across alive pixels and at last go to outside of the PMT matrix and TUS FoV. The EAS movement is shown by arrows and its exit outside of FoV may be a possible reason for the Cherenkov peak absence at the EAS end.

The next step of the EAS candidate study is a reconstruction of its arrival directions. It is possible to do after the relative calibration coefficient calculated. Some details of the event reconstruction procedure and arrival directions measurements are presented in [4]. To check a correctness and reliability of the procedure, the same analysis was fulfilled for MC simulated events that is generated with ESAF package [7] and the TUSSIM program [8]. Events modeled by ESAF were analyzed in the same way as real events. A difference between the arrival angles of simulated EAS as given by ESAF program generator and the reconstructed zenith $\theta$ and azimuth $\varphi$ angles for these events is presented in Figure 4. It gives an evaluation of the systematic errors of these angles measurements. There are rather long tails in the differences those are not exactly understood. Possible reasons: 1) presently the TUSFitData program doesn't take into account that TUS detector is out of vertical plane with EAS axes, 2) ESAF generated arrival angles doesn't affected by off axis EAS Cherenkov peak but the reconstructed ones do it. 

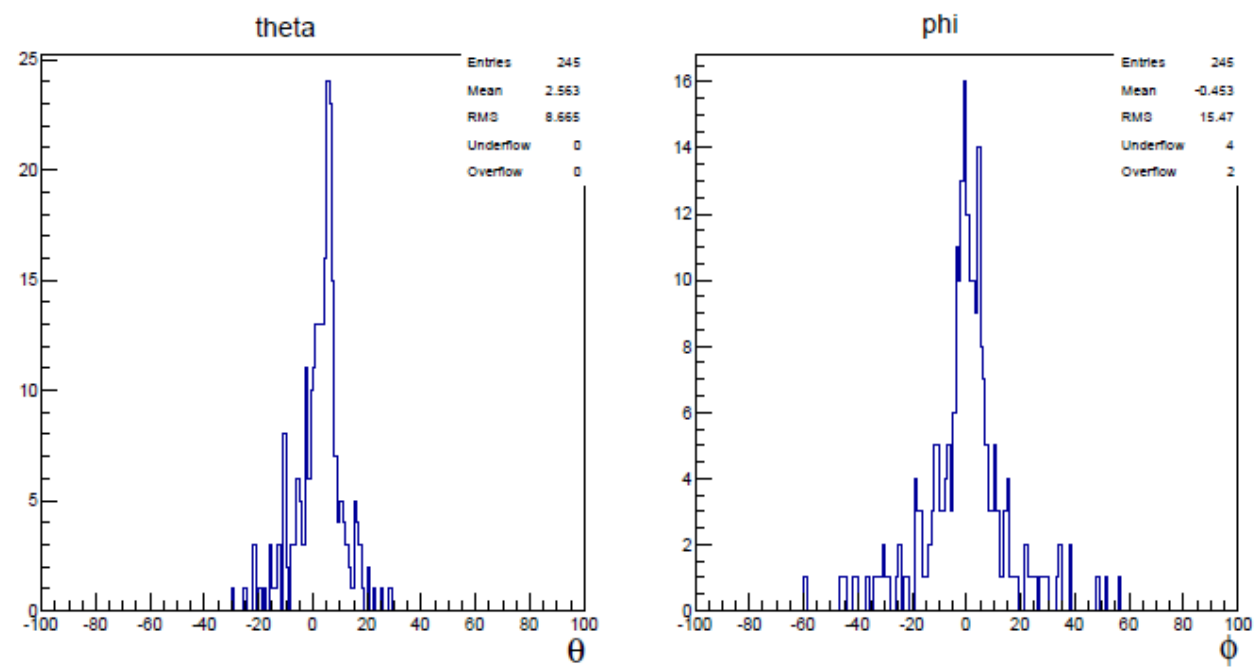

Figure 4: Difference between ESAF simulated and TUSReadData reconstructed zenith $\theta$ and azimuth $\varphi$ angles.

To estimate the statistical errors of arrival angles of the measured EAS events, location of the hit coordinates were randomly variated inside of every pixel at a particular point in time. Afterwards the linear 3D-fit of hit pixels was done and arrival angles calculated by the reasonable number times with a such randomization and real amplitudes of the pixels. The distributions of $\theta$ and $\varphi$ angles are plotted on the histograms and are presented in Figure 5. Variation of the minimum threshold value for the amplitude (in ADC codes) leads to inessential change the angles and presented in Table 1.
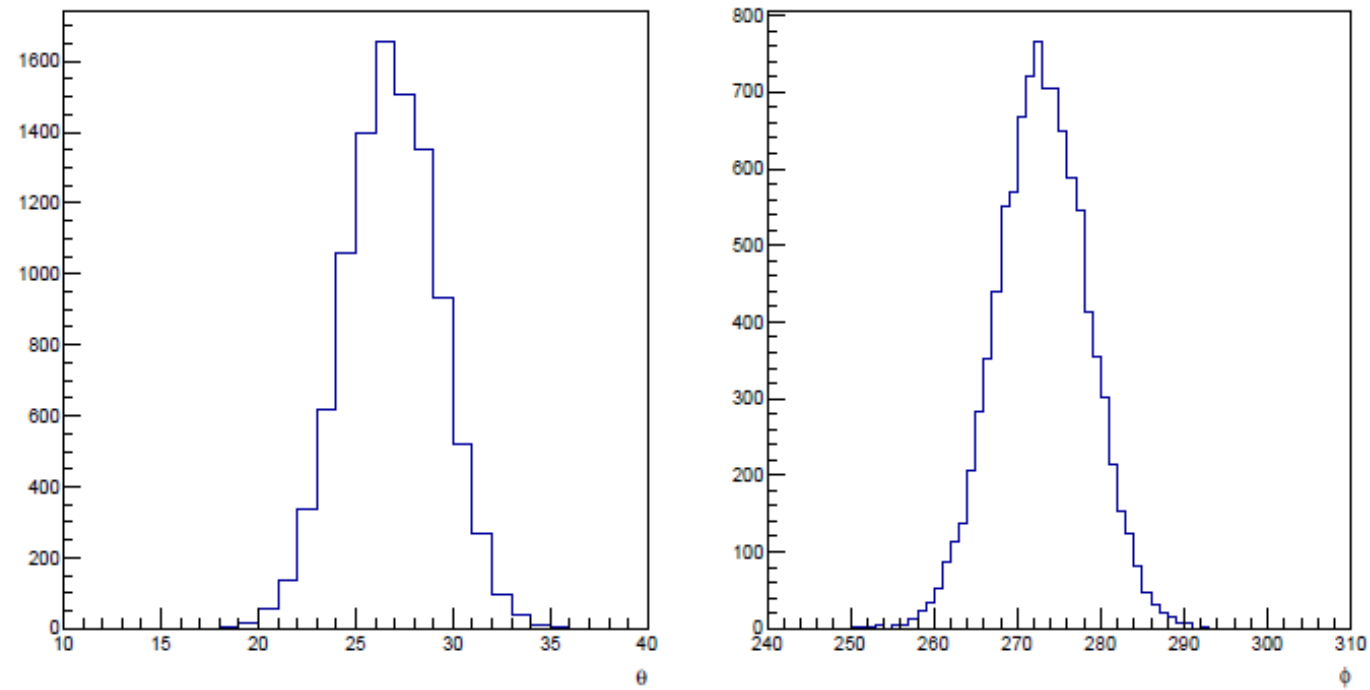

Figure 5: Distributions of the $\theta$ and $\varphi$ angles measurements for event \#487. 


\begin{tabular}{|l|ll|ll|}
\hline threshhold & $\# 487$ & & $\# 123$ \\
& $\theta$ & $\phi$ & $\theta$ & $\phi$ \\
\hline 0 & $29 \pm 2$ & $279,3 \pm 4,5$ & $55,82 \pm 2,4$ & $234,6 \pm 2,8$ \\
5 & $27,9 \pm 2,2$ & $273 \pm 5,2$ & $54,17 \pm+2,4$ & $233 \pm 3$ \\
10 & $27,6 \pm 2,4$ & $295,6 \pm 5,3$ & $52,7 \pm 3$ & $227,7 \pm 3,6$ \\
15 & $27,6 \pm 2,4$ & $295,8 \pm 5$ & $42,9 \pm 4,2$ & $218,7 \pm 6,3$ \\
\hline
\end{tabular}

Table 1: Dependence of the angles $\theta$ and $\varphi$ from the variation of the minimum threshold value for the amplitude (in ADC codes)

Finally the following arrival angles were obtained for the EAS candidates: $\theta \approx$ $28^{\circ} \pm 2.5^{\circ}{ }_{\text {stat }} \pm 8^{\circ}{ }_{\text {syst }}, \varphi \approx 275^{\circ} \pm 5^{\circ}{ }_{\text {stat }} \pm 8^{\circ}$ syst for event $\# 487$ and $\theta \approx 54^{\circ} \pm 2: 5^{\circ}{ }_{\text {stat }} \pm 8^{\circ}{ }_{\text {syst }} \varphi \approx 233^{\circ}$ $\pm 4^{\circ}$ stat $\pm 8^{\circ}$ syst for event \#123 in the TUS coordinate system. For a more correct assessment of the angles, a $3 \mathrm{~d}$ fit program is currently being developed. Analysis of TUS data shows that most of the events cannot be the EAS candidates. It looks like a short $\sim 150 \mu$ s flash of light. Typically such events look like tracks of the few pixels length. The zenith angles of such events are near zero. It means that we have a non-moving flash source of light. There were no Cherenkov flashes at the ends of cascade curves as it may be expected in EAS events. Besides the time durations of the signals are longer than it should be for vertical EASs. The pseudo EAS event's distribution on the PMT matrix is homogeneous that excludes the apparatus nature for these events. The majority of EAS-like events can be related to fast anthropogenic signals.

\section{Discussion and conclusion}

The TUS detector is operating on board the "Lomonosov" satellite. TUS proved the possibility of registration of UHECR from the space orbit. During TUS data taking in EAS mode and a search for an UHECR a large number of rapid events of the various origins were observed that take place in the atmosphere of the Earth. At least two EAS candidates were selected and their arrival angles were measured. The EAS candidate energy evaluation is not yet obtained due to absence of the absolute PMT calibration. Analysis of TUS data shows that most of the events cannot be the EAS candidates [4]. Nevertheless some of these events may contain genuine EAS events at energy $>70 \mathrm{EeV}$ that is lower limit of the sensitivity according to TUS detector simulation [4]. A more detailed analysis of the TUS data is in progress for search of the other EAS candidate events.

\section{Acknowledgements}

The TUS experiment on board the "Lomonosov" satellite was realized within the Federal Space Program of Russia with funding by the Russian Space Agency. The data analysis is supported by RFBR grants No. No. 15-02-05498. 


\section{References}

[1] RG. K. Garipov et al., Bulletin of the Russian Academy of Sciences: Physics, V79 Issue 3, 326-328, (2015)

[2] The JEM-EUSO Collaboration, Experimental Astronomy, V40 Issue 1, 19-44, (2015)

[3] P. A. Klimov et al., Space Science Reviews, V212 Issue 3-4, 1687-1703 (2017)

[4] L.G.Tkachev. PoS ICRC2017 527 (2018)

[5] B.A. Khrenov at al., Journal of Cosmology and Astroparticle Physics V2017 Issue 09, 006 (2017)

[6] S.V. Biktemerova et al., arXiv:1706.05369v1 [astro-ph.IM] 16 Jun 2017

[7] C. Berat, S. et al., Astroparticle Physics, 33, 221-247, (2010)

[8] A. Grinyuk et al., Astroparticle Physics 90 93-97 (2017) 